\title{
Kournal of Biomedicine
}

2017; 2: 39-44. doi: 10.7150/jbm.17983

Research Paper

\section{A macroscopic analysis of the epidemiology of thoracic aorta atherosclerosis: An autopsy study}

\author{
Apostolos S. Gogakos ${ }^{1,2}$, Fotios Chatzinikolaou1,2, Triantafyllia Koletsa ${ }^{3}$, Leonidas Pavlidis ${ }^{4}$, Dimitrios \\ Paliouras ${ }^{2}$, Thomas Rallis², Nikolaos Schizas 2 , Paul Zarogoulidis ${ }^{5}$, Georgia Trakada6 ${ }^{6}$ Christos Simoglou , \\ Nikolaos Katsikogiannis7, Eirini Sarika7, Achilleas Lazopoulos², Ilias Karapantzos ${ }^{8}$, Pavlos Sarafis9, \\ Nikolaos Barbetakis² \\ 1. Department of Forensic Medicine \& Toxicology, Faculty of Medicine, Aristotle University of Thessaloniki, Thessaloniki, Greece; \\ 2. Thoracic Surgery Department, Theagenio Cancer Hospital, Thessaloniki, Greece; \\ 3. Department of Pathology, Faculty of Medicine, Aristotle University of Thessaloniki, Thessaloniki, Greece; \\ 4. Department of Plastic and Reconstructive Surgery, Faculty of Medicine, Aristotle University of Thessaloniki, Thessaloniki, Greece; \\ 5. Pulmonary Department-Oncology Unit, "G. Papanikolaou” General Hospital, Aristotle University of Thessaloniki, Thessaloniki, Greece; \\ 6. Division of Pulmonology, Department of Clinical Therapeutics, National and Kapodistrian University of Athens School of Medicine, Alexandra Hospital, \\ Athens, Greece; \\ 7. Surgery Department (NHS), University General Hospital of Alexandroupolis, Alexandroupolis, Greece; \\ 8. Ear, Nose and Throat Department, "Saint Luke" Private Hospital, Panorama, Thessaloniki, Greece; \\ 9. Department of Nursing, School of Health Sciences, Cyprus University of Technology, Limassol, Cyprus.
}

Corresponding author: Paul Zarogoulidis, MD. PhD. Pulmonary Department-Oncology Unit, "G. Papanikolaou” General Hospital, Aristotle University of Thessaloniki, Thessaloniki, Greece. Fax: 00302310992424 Mobile: 00306977271974 E-mail: pzarog@hotmail.com.

(c) Ivyspring International Publisher. This is an open access article distributed under the terms of the Creative Commons Attribution (CC BY-NC) license (https://creativecommons.org/licenses/by-nc/4.0/). See http://ivyspring.com/terms for full terms and conditions.

Received: 2016.10.18; Accepted: 2016.12.06; Published: 2017.02.03

\begin{abstract}
Background: Atherosclerosis is the most important cardiovascular disease across the globe with a large proportion of associated morbidity and mortality. The aim of this study is to detect the presence of atherosclerosis-like lesions in the three segments of the thoracic aorta (ascending aorta, aortic arch and descending aorta) of 50 unselected adult cadavers of all ages. Methods: An autopsy analysis was performed using 50 unselected adult cadavers ( 37 male, 13 female - mean age 64.2 years; age range 19-90 years). Macroscopic dissection of the three segments of the thoracic aorta was performed and the presence of atherosclerosis-like lesions was evaluated according to the subjects' demographic and clinical characteristics. Results: Atherosclerotic lesions in at least one of the three aortic segments were identified in $82 \%$ of cadavers, $83.78 \%$ of male and $76.92 \%$ of female. Of 50 subjects, $30 \%$ had an atherosclerotic lesion in the ascending aorta, $48 \%$ in the aortic arch and $44 \%$ in the descending aorta. No aneurysmal changes were detected. Smoking, hypertension, diabetes, hypercholesterolemia, obesity and coronary disease were the risk factors correlated most with the presence of atherosclerosis. Conclusions: Atherosclerosis-like lesions in the thoracic aorta are prevalent in adults of all ages. Obesity is a major risk factor regarding the development of atheromas. This cadaveric study comes to underline the necessity of further similar studies in order to evaluate the presence of atherosclerosis in the thoracic aorta, and also helps to assess the risk of major cardiovascular events in the general population.
\end{abstract}

Key words: Atherosclerosis, Autopsy, Thoracic aorta, Cadaver, Macroscopic.

\section{Introduction}

Atherosclerosis is a chronic inflammatory disease and is responsible for over $50 \%$ of all mortality in Western populations (1). It occurs in the elastic and muscular arteries and involves the vascular cells, the immune system and several organs (2). Immune responses, both adaptive and innate, participate in 
every phase of atherosclerosis. These mechanisms result in an inflammatory response, which attacks the endothelium and smooth muscle of the artery wall, causing major cardiac and cerebral complications (3).

The association between aortic atherosclerosis and other major cardiovascular diseases is quite significant. Atherosclerosis in the thoracic aorta is closely related to the degree of coronary and carotid disease (4). The most common risk factors that may inhibit or accelerate atherosclerosis are hyperlipidemia, smoking, hypertension and diabetes (5).

The main purpose of this study is to macroscopically examine the presence of atherosclerosis-like lesions in the thoracic aortas of 50 cadavers and underline its significance in correlation with the demographic and clinical characteristics of the population.

\section{Methods}

The study was carried out on thoracic aortas obtained during routine autopsies of 50 cadavers in the Laboratory of Forensic medicine and Toxicology of the Aristotle University of Thessaloniki, Greece during a period of one year. The cause of death was obtained from death certificates (Table 1). Inclusion criteria were age over 18 years and an autopsy had to be intended. A signed letter of consent was taken from the relatives of the dead bodies. Where consensus could not be reached, third independent review was sought from a Professor of Forensic medicine.

Table 1. Demographic data and cause of death.

\begin{tabular}{llll}
\hline Cause of Death & $\mathbf{n}(\mathbf{\%}) \mathbf{N = 5 0}$ & Age in years (mean) & $\mathbf{M} / \mathbf{F}$ \\
\hline MI & $19 / 50(38 \%)$ & 66.1 & $16 / 3$ \\
Accident & $18 / 50(36 \%)$ & 57.1 & $13 / 5$ \\
Cancer & $11 / 50(22 \%)$ & 67.2 & $6 / 5$ \\
COPD & $2 / 50(4 \%)$ & 67 & $2 / 0$ \\
\hline
\end{tabular}

MI, myocardial infarction; COPD, chronic obstructive pulmonary disease; $\mathrm{F}$, female; M, male.

After explanation from the corpse, the thoracic aortas were photographed and washed in water without further fixation or staining. Each thoracic aorta was incised longitudinally and was examined macroscopically. Three $5 \mathrm{~mm}$ tissue samples from each aorta were obtained for analysis from three standard anatomic stations (Figure 1), which are considered to be more fragile and favor the development of atherosclerotic plaques, according to literature (6-11). These stations were the anterior wall of the ascending aorta, the intimal surface of the aortic arch (Figure 2a) and the point of the descending aorta just below the origin of the subclavian artery (Figure 2b).

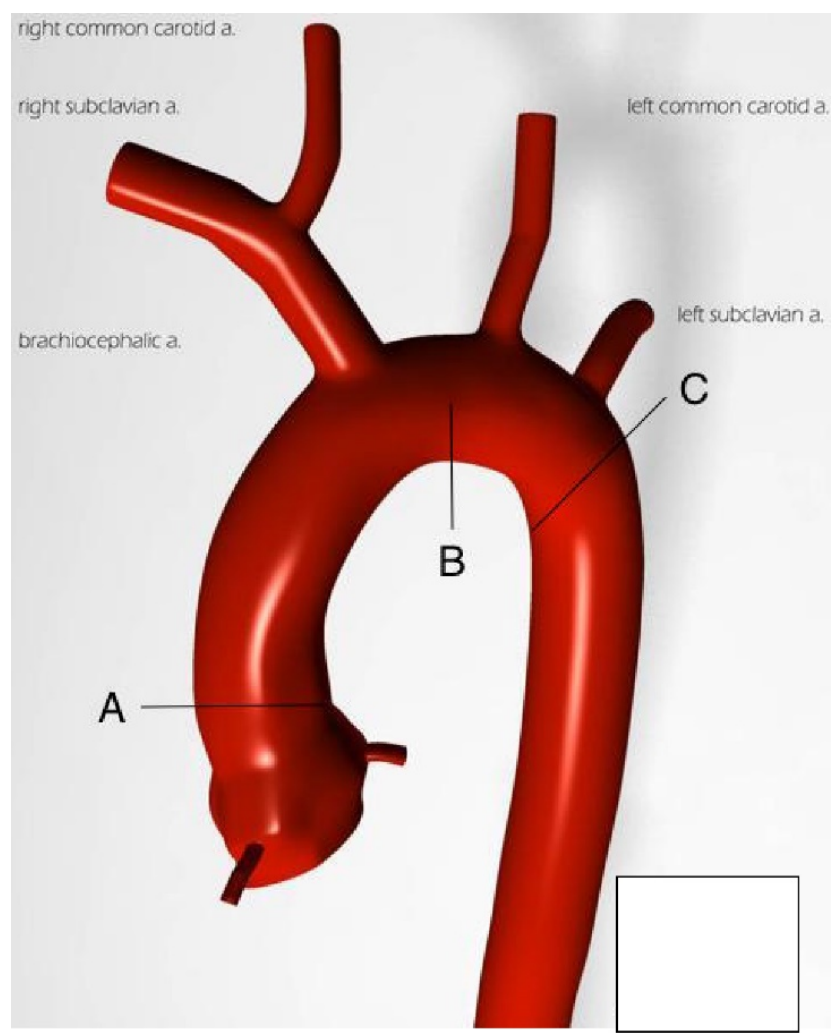

Figure 1. Schematic illustration of the sites of specimen collection. A: ascending aorta; B: aortic arch; C: descending aorta (30). 


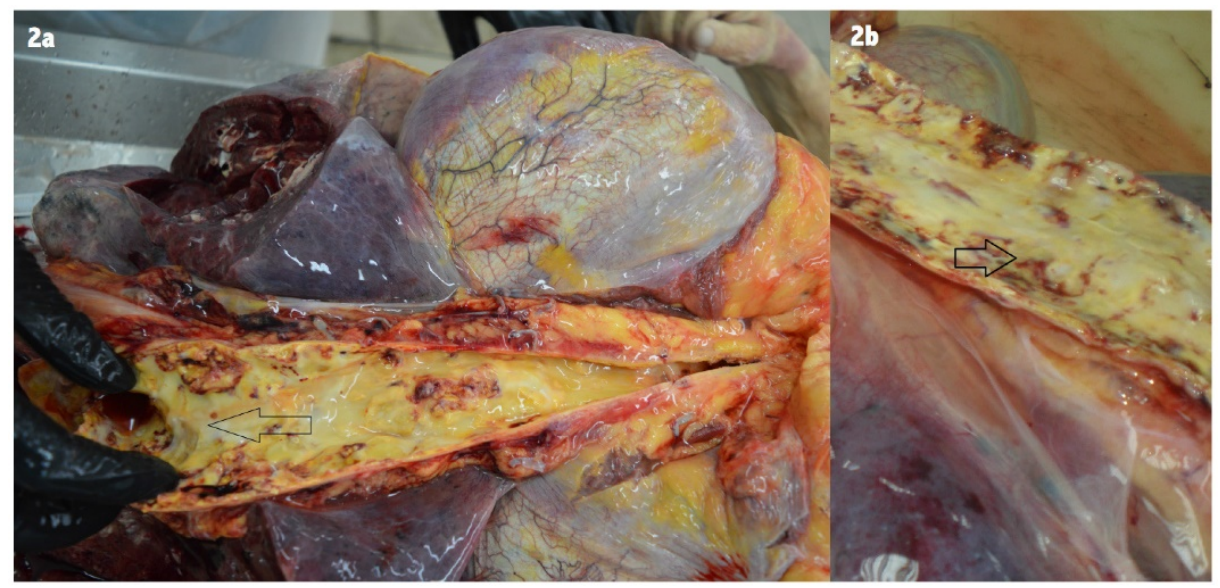

Figure 2. (a) Anatomic sample of the anterior wall of the ascending aorta and the intimal surface of the aortic arch. (b) Anatomic sample of the descending aorta just below the origin of the subclavian artery.

Specimens were examined for the presence and location of atherosclerosis-like lesions. Demographic characteristics (age, gender), season and location of death were recorded. Smoking, alcohol, hypertension, diabetes, coronary disease, hypercholesterolemia and body mass index (BMI) were evaluated in correlation with the presence of atherosclerosis.

This study was approved by the local ethics committee and was carried out in accordance with the World Medical Association's Declaration of Helsinki.

All analyses were performed with SPSS 21 software. Statistics are reported as mean and SD, median with interquartile range or counts (\%). Normality test have been done and variables were compared with the parametric $t$-test or the nonparametric Mann-Whitney U test, as appropriate. For determination of the relationship between the biological variables and the presence of atheromas, Pearson correlation coefficients were computed and multiple regression analysis was performed.

\section{Results}

An autopsy analysis was performed using 50 unselected adult cadavers ( 37 male, 13 female - mean age 64.2 years; age range $19-90$ years). The clinical characteristics of the study subjects are shown in Table 2.

Atherosclerotic lesions in at least one of the three aortic segments were identified in $82 \%$ of cadavers (41/50), $83.78 \%$ of male and $76.92 \%$ of female. The most common site of atherosclerosis was identified in the aortic arch, with a percentage of $48 \%(24 / 50)$, followed by the descending aorta $(44 \%-22 / 50)$ and the ascending aorta $(30 \%-15 / 50)$.

Age proved to be an important risk factor for developing atherosclerosis, especially in the ascending aorta, with a negative correlation of -0.35 , as $8 / 15$ subjects $(53.33 \%)$ were less than 65 years old (Table 3).

Table 2. Clinical characteristics and macroscopic atherosclerosis-like lesions according to site.

\begin{tabular}{lccc}
\hline & $\begin{array}{c}\text { Ascending } \\
\text { Aorta (n=15) }\end{array}$ & $\begin{array}{l}\text { Aortic Arch } \\
(\mathbf{n = 2 4})\end{array}$ & $\begin{array}{c}\text { Descending } \\
\text { Aorta (n=22) }\end{array}$ \\
\hline Men/Women & $13 / 2$ & $14 / 10$ & $18 / 4$ \\
Age in years (mean) & 65.13 & 68.25 & 67.72 \\
Smoking & 10 & 16 & 15 \\
Alcohol & 2 & 4 & 4 \\
Hypertension & 8 & 17 & 14 \\
CD & 5 & 9 & 8 \\
Diabetes & 4 & 5 & 7 \\
Hypercholesterolemia & 5 & 11 & 10 \\
\hline
\end{tabular}

Table 3. Number of subjects with atherosclerosis-like lesions according to site and age.

\begin{tabular}{lccc}
\hline $\begin{array}{l}\text { Age group } \\
\text { (years) } \mathbf{N = 5 0}\end{array}$ & $\begin{array}{c}\text { Ascending Aorta } \\
(\mathbf{n}=\mathbf{1 5})\end{array}$ & $\begin{array}{c}\text { Aortic Arch Descending Aorta } \\
(\mathbf{n}=\mathbf{2 4})\end{array}$ & \begin{tabular}{c}
$(\mathbf{n}=\mathbf{2 2})$ \\
\hline$<65(18 / 25)$
\end{tabular} \\
\hline$<$ & 9 & 10 \\
$>65(23 / 25)$ & 7 & 15 & 12 \\
\hline
\end{tabular}

Smoking had a vast impact in the presence of atherosclerosis across the thoracic aorta, with a negative correlation of -0.19 for the ascending aorta, -0.77 for the aortic arch, and -0.83 for the descending aorta, as $34 / 50$ subjects were smokers $(68 \%)$.

$28 / 50$ specimens had a history of hypertension (56\%), with $8 / 15$ and $13 / 22$ presented with atherosclerotic lesions in the ascending and descending aorta respectively (Pearson correlation: -0.35 and 0.55 respectively). 
$19 / 50$ subjects (38\%) suffered from coronary artery disease, which was highly correlated with the presence of atherosclerosis in the ascending and descending aorta (Pearson correlation: -0.63 and 0.30 respectively).

Diabetes was another risk factor that was taken under consideration $(12 / 50-24 \%)$, and was strongly correlated with the development of atherosclerosis in the ascending aorta. (Pearson correlation: 0.41).

$17 / 50$ subjects $(34 \%)$ were identified with atherosclerosis-like lesions in more than one anatomic stations of the thoracic aorta. Furthermore, 10/17 subjects with multiple atherosclerotic lesions (58.9\%) did not receive any statin therapy for hypercholesterolemia (Pearson correlation: -0.89 ascending aorta, 0.15 - aortic arch, 0.99 - descending aorta).

As shown in Table 4 , subjects $<65$ years old had a mean BMI of $25.76 \mathrm{~kg} / \mathrm{m}^{2}$, while in subjects $>65$ years old, the mean BMI was $28.71 \mathrm{~kg} / \mathrm{m}^{2}$, indicating that most subjects were overweight. Furthermore, BMI had a significant correlation with the presence of atheromas in all three segments of the thoracic aorta (Pearson correlation: 0.79 - ascending aorta, 0.35 aortic arch, 0.21 - descending aorta).

Table 4. Anthropometric characteristics of subjects in the current study (mean $\pm S D$ ).

\begin{tabular}{llll}
\hline $\begin{array}{l}\text { Age group (years) } \\
\mathbf{N = 5 0}\end{array}$ & $\begin{array}{l}\text { Body height } \\
\text { (cm) }\end{array}$ & $\begin{array}{l}\text { Body weight } \\
\mathbf{( k g )}\end{array}$ & $\begin{array}{l}\text { BMI } \\
\mathbf{( k g / \mathbf { m } ^ { 2 } )}\end{array}$ \\
\hline$<65$ & $174 \pm 5$ & $78 \pm 8$ & $25.76 \pm 3.12$ \\
$>65$ & $169 \pm 4$ & $82 \pm 6$ & $28.71 \pm 2.01$ \\
\hline
\end{tabular}

BMI, body mass index.

\section{Discussion}

Atherosclerosis is a chronic inflammatory process, which involves the entire arterial vasculature $(2,12)$. The aorta is affected earliest, followed by the carotid and coronary arteries (13). While the etiology of atherosclerosis is unknown, several factors have been described over the decades that contribute to atherosclerotic plaque progression. In 1999, Ross et al stated that atherogenic actions are triggered from metabolic and inflammatory stress factors, which include lipid modification in the intima, oxidative stress and prothrombotic stimuli (14). Thus, cytokines, chemokines and growth factors seem to play a significant part in the inflammatory process $(14,15)$. However, Amiya et al in 2014 indicated that vascular function could also be impaired from alterations in the autonomic nervous system (16).
In human beings, atherosclerosis is the most common pathologic process leading to several major cardiac and vascular diseases (17). Particularly, thoracic aortic atherosclerosis is responsible for most myocardial infarctions, strokes and aneurisms $(4,15,18,19,20)$.

An autopsy study can offer to the researcher the basic knowledge of understanding the essentials of atherosclerosis and its clinical significance. The objective, therefore, of this study was to macroscopically examine the presence of atherosclerosis-like lesions in the thoracic aortas of 50 cadavers and underline its significance in correlation with the demographic and clinical characteristics of the population.

Age is a major risk factor for cardiovascular diseases. The thoracic aorta dimensions alter with aging and deserve further attention in order to prevent aortic aneurisms (20). In this study, no aneurisms were detected, while age was strongly correlated with the presence of atherosclerotic-like lesions in the ascending aorta.

It has been generally accepted that anthropometric characteristics, such as high body weight, weight gain and increased BMI are crucial risk factors for the development of cardiovascular diseases (21). Schreyer et al reported that apolipoprotein receptors could have an immense effect on developing atherosclerosis associated with obesity (22). These findings are in total agreement with the current study, indicating that obesity is indeed an important risk factor in the development of atherosclerosis.

The distribution of atherosclerosis varies by segments of the aorta. A population-based transesophageal echocardiography (TEE) study by Agmon et al on thoracic atherosclerosis reported that the ascending aorta had the lowest prevalence of atheroma (19), which is in agreement with the findings of the current study, as $30 \%$ of the cadavers had atherosclerotic-like lesions in this particular segment. A possible explanation for such event might be the fact that the ascending aorta has no branch vessels. Most atheromas occur at sites where shear stress is low, yet fluctuating, such as branch vessels or sites where vessel diameter changes (23).

This autopsy report comes to underline the lack of similar studies in literature, regarding the anatomy and the presence of atheromas in the thoracic aorta and its three segments in particular. Several studies have been published, though using only imaging methods and protocols in order to evaluate the mechanisms that cause atherosclerosis. Moreover, cadaveric studies regarding the presence of 
atherosclerosis are also found in literature. However, their main goal was to investigate atheromas in different sites of the cardiovascular system, such as the coronary arteries and/or the aortic valve (24-26).

Regarding the epidemiology of atherosclerosis in Greece, few studies can be found in literature. A retrospective study by Barbetseas et al in 2008 used TEE to compare the degree of atherosclerosis of the aorta in patients with type A and type B acute thoracic aortic dissection. They resulted that there was a significant association between the presence of severe atherosclerosis and Type B dissection, as well as hypertension. In addition, the presence of atheromas in the ascending aorta was less frequent compared to the other two segments. These findings are in total agreement with the results of the present study (27). Moreover, in 2000, a study by Kallikazaros et al that examined the relation between carotid and ascending aortic atherosclerosis in cardiac patients using again TEE, indicated that a higher percentage of patients with aortic atherosclerotic plaques suffered from hypertension and diabetes mellitus compared with patients without aortic plaques (28). A more recent study by Kostopoulos et al in 2014 examined the possible role of local chemerin and CMKLR1 expression in atherosclerosis using coronary arteries and abdominal aortas from 40 cadavers. Hypertension was again strongly correlated with the expression of chemerin in both sites (29).

In conclusion, thoracic aortic atherosclerosis is accounted for many cardiac and vascular diseases. Age and obesity have proved to be important risk factors in the development of atherosclerosis. An autopsy study of the thoracic aorta can provide the most accurate results to a better understanding of its anatomy as well as the incidence of atherosclerotic-like lesions in its segments. This cadaveric study comes to underline the necessity of further similar studies in order to evaluate the presence of atherosclerosis in the thoracic aorta, and also helps to assess the risk of major cardiovascular events in the general population. To our knowledge this is the first cadaveric study that macroscopically examines the presence of atherosclerosis-like lesions in the thoracic aorta in correlation with the demographic and clinical characteristics of the Greek population.

\section{Limitations of the study}

This study is consisted of a moderate number of autopsy subjects in a population with a high prevalence of atherosclerosis, causing limitations in both the external and the internal validity of the study. Moreover, the results may not reflect the precise prevalence of thoracic aortic atherosclerosis in other western populations, including Greece. Large scale studies, therefore, are needed to confirm the presence of atherosclerotic-like lesions in the thoracic aorta in different populations.

\section{Competing Interests}

The authors have declared that no competing interest exists.

\section{References}

1. Ross R. The pathogenesis of atherosclerosis: A perspective for the 1990s. Nature 1993;362(6423):801-809.

2. De Tena JG, Kriszbacher I, Koppán M, Bódis J, Hansson GK. Inflammation, atherosclerosis, and coronary artery disease [5] (multiple letters). New Engl J Med 2005;353(4):429-430.

3. Hansson GK, Libby P. The immune response in atherosclerosis: A double-edged sword. Nat Rev Immunol 2006;6(7):508-519.

4. Tresoldi S, Di Leo G, Zoffoli E, Munari A, Primolevo A, Cornalba G, et al. Association of aortic wall thickness on contrast-enhanced chest CTwith major cerebro-cardiac events. Acta Radiol 2014;55(9):1040-1049.

5. Youssef G, Guo M, McClelland RL, Shavelle DM, Nasir K, Rivera J, et al. Risk Factors for the Development and Progression of Thoracic Aorta Calcification: The Multi-Ethnic Study of Atherosclerosis. Acad Radiol 2015;22(12):1536-1545.

6. Crawford ES, Crawford JL. Diseases of the Aorta. Including an Atlas of Angiographic Pathology and Surgical Technique. Williams and Wilkins, 1984.

7. Kirklin J, Barratt-Boyes B. Cardiac Surgery, 2nd ed. New York, Churchill-Livingstone, 1993.

8. Crawford ES, Saleh SA. Transverse aortic arch aneurysm. Improved results of treatment employing new modifications of aortic reconstruction and hypothermic cerebral circulatory arrest. Ann Surg 1981;194(2):180-188.

9. Yacoub MH, Kilner PJ, Birks EJ, Misfeld M. The aortic outflow and root: A tale of dynamism and crosstalk. Ann Thorac Surg 1999;68(3 SUPPL.):S37-S43.

10. Dare AJ, Veinot JP, Edwards WD, Tazelaar HD, Schaff HV. New observations on the etiology of aortic valve disease: A surgical pathologic study of 236 cases from 1990. Hum Pathol 1993;24(12):1330-1338.

11. Lung B, Baron G, Butchart EG, Delahaye F, Gohlke-Bärwolf C, Levang $\mathrm{OW}$, et al. A prospective survey of patients with valvular heart disease in Europe: The Euro Heart Survey on valvular heart disease. Eur Heart J 2003;24(13):1231-1243.

12. Nichols WW, O'Rourke MF, Avolio AP, Yaginuma T, Murgo JP, Pepine $\mathrm{CJ}$, et al. Effects of age on ventricular-vascular coupling. Am J Cardiol 1985;55(9):1179-1184.

13. Sawabe M, Arai T, Kasahara I, Hamamatsu A, Esaki Y, Nakahara K-, et al. Sustained progression and loss of the gender-related difference in atherosclerosis in the very old: A pathological study of 1074 consecutive autopsy cases. Atherosclerosis 2006;186(2):374-379.

14. Ross R, Dodet B. Atherosclerosis is an inflammatory disease. Am Heart J 1999;138(5 II):S419-S420.

15. Libby P. Inflammation in atherosclerosis. Nature 2002;420(6917):868-874.

16. Amiya E, Watanabe M, Komuro I. The Relationship between Vascular Function and the Autonomic Nervous System. Ann Vasc Dis. 2014;7(2):109-119.

17. Willecke F, Yuan C, Oka K, Chan L, Hu Y, Barnhart S, et al. Effects of high fat feeding and diabetes on regression of atherosclerosis induced by low-density lipoprotein receptor gene therapy in LDL receptor-deficient mice. PLoS ONE 2015;10(6)

18. Cohen A. Atherosclerosis of the Thoracic Aorta. Further Characterization for Higher Risk of Vascular Events ${ }^{* *}$ Editorials published in the Journal of the American College of Cardiology reflect the views of the authors and do not necessarily represent the views of JACC or the American College of Cardiology. J Am Coll Cardiol 2008;52(10):862-864.

19. Agmon Y, Khandheria BK, Meissner I, Schwartz GL, Petterson TM, O'Fallon WM, et al. Independent association of high blood pressure and aortic atherosclerosis: A population-based study. Circulation 2000;102(17):2087-2093. 
20. Effects of aging on thoracic aorta size and shape: A non-contrast CT study. Proceedings of the Annual International Conference of the IEEE Engineering in Medicine and Biology Society, EMBS; 2012.

21. Rexrode KM, Hennekens CH, Willett WC, Colditz GA, Stampfer MJ, Rich-Edwards JW, et al. A prospective study of body mass index, weight change, and risk of stroke in women. J Am Med Assoc 1997;277(19):1539-1545.

22. Schreyer SA, Lystig TC, Vick CM, LeBoeuf RC. Mice deficient in apolipoprotein $\mathrm{E}$ but not LDL receptors are resistant to accelerated atherosclerosis associated with obesity. Atherosclerosis 2003;171(1):49-55.

23. Ku DN, Giddens DP, Zarins CK, Glagov S. Pulsatile flow and atherosclerosis in the human carotid bifurcation. Positive correlation between plaque location and low and oscillating shear stress. Arteriosclerosis 1985;5(3):293-302.

24. Abedinzadeh N, Pedram B, Sadeghian Y, Nodushan SMHT, Gilasgar M, Darvish M, et al. A histopathological analysis of the epidemiology of coronary atherosclerosis: An autopsy study. Diagn Pathol 2015;10(1).
25. Kuusisto J, Räsänen K, Särkioja T, Alarakkola E, Kosma V-. Atherosclerosis-like lesions of the aortic valve are common in adults of all ages: A necropsy study. Heart 2005;91(5):576-582.

26. Otto CM, Kuusisto J, Reichenbach DD, Gown AM, O'Brien KD. Characterization of the early lesion of 'degenerative' valvular aortic stenosis: Histological and immunohistochemical studies. Circulation 1994;90(2):844-853.

27. Barbetseas J, Alexopoulos N, Brili S, Aggeli C, Chrysohoou C, Frogoudaki A, et al. Atherosclerosis of the aorta in patients with acute thoracic aortic dissection. Circ J 2008;72(11):1773-1776.

28. Kallikazaros IE, Tsioufis CP, Stefanadis CI, Pitsavos CE, Toutouzas PK. Closed relation between carotid and ascending aortic atherosclerosis in cardiac patients. Circulation 2000;102(19)

29. Kostopoulos CG, Spiroglou SG, Varakis JN, Apostolakis E, Papadaki $\mathrm{HH}$. Chemerin and CMKLR1 expression in human arteries and periadventitial fat: A possible role for local chemerin in atherosclerosis? BMC Cardiovasc Disord 2014;14.

30. Frank Gaillard. Case courtesy of Prof Frank Gaillard, Radiopaedia.org, rID: 35949. 